$$
\begin{aligned}
& 2000-G T-0194 \\
& \text { ASNE Turbo } 2000 \\
& \text { Munich, Germany, Bay z-11. }
\end{aligned}
$$

\title{
HEAT TRANSFER AND PRESSURE DISTRIBUTIONS ON A GAS TURBINE BLADE TIP
}

\author{
Gm S. Azad, Je-Chin Han, Shuye Teng \\ Turbine Heat Transfer Laboratory \\ Department of Mechanical Engineering \\ Texas A\&M University \\ College Station, Texas 77843-3123
}

Robert J. Boyle
NASA Glenn Research Center, Cleveland, Ohio 44135

\begin{abstract}
Heat transfer coefficient and static pressure distributions are experimentally investigated on a gas turbine blade tip in a five-bladed stationary linear cascade. The blade is a 2-dimensional model of a first stage gas turbine rotor blade with a blade tip profile of a GE-E ${ }^{3}$ aircraft gas turbine engine rotor blade. The flow condition in the test cascade corresponds to an overall pressure ratio of 1.32 and exit Reynolds number based on axial chord of $1.1 \times 10^{6}$. The middle 3-blade has a variable tip gap clearance. All measurements are made at three different tip gap clearances of about $1 \%, 1.5 \%$, and $2.5 \%$ of the blade span. Heat transfer measurements are also made at two different turbulence intensity levels of $6.1 \%$ and $9.7 \%$ at the cascade inlet. Static pressure measurements are made in the mid-span and the near-tip regions as well as on the shroud surface, opposite the blade tip surface. Detailed heat transfer coefficient distributions on the plane tip surface are measured using a transient liquid crystal technique. Results show various regions of high and low heat transfer coefficient on the tip surface. Tip clearance has a significant influence on local tip heat transfer coefficient distribution. Heat transfer coefficient also increases about $15-20 \%$ along the leakage flow path at higher turbulence intensity level of $9.7 \%$ over $6.1 \%$.
\end{abstract}

\section{NOMENCLATURE}

C Tip clearance gap

$C_{p}$ Coefficient of pressure

$C_{x}$ Axial chord length of the blade $(8.61 \mathrm{~cm})$

$\mathrm{h}$ Local convective heat transfer coefficient $\left(\mathrm{W} / \mathrm{m}^{2} \mathrm{~K}\right)$

k Thermal conductivity of blade tip material $(0.18 \mathrm{~W} / \mathrm{m} \mathrm{K})$

LE Leading edge of the blade

$P$ Local static pressure

$P_{\text {avg }}$ Averaged static pressure

$P_{t}$ Total pressure at the inlet

P/S Pressure side of the blade

S/S Suction side of the blade

$t$ Transition time for liquid crystal color change

TE Trailing edge of the blade
$T_{i}$ Initial temperature of the blade tip surface

$T_{\infty}$ Mainstream temperature of the flow

$T_{w}$ Color change temperature of the liquid crystal, green-to-red

Tu Turbulence intensity level at the inlet

$\mathrm{X}$ Axial distance $(\mathrm{cm})$

$\alpha \quad$ Thermal diffusivity of tip material $\left(1.25 \times 10^{-7} \mathrm{~m}^{2} / \mathrm{s}\right)$

\section{INTRODUCTION}

A recent trend in modem gas turbine engines is to increase the combustor outlet temperature to achieve higher thermal efficiency and higher power output. This increase in temperature is detrimental to the downstream first stage turbine blades. Therefore, a proper combination of aerodynamics, heat transfer, and material capabilities is required to design a high efficiency gas turbine engine. Many research efforts have been dedicated to developing efficient aerodynamics and cooling techniques for turbine airfoils. However, some critical regions still remain that require more frequent inspection and repair due to frequent breakdowns. Blade tip and near-tip regions fall into this category. Blade tip and near-tip regions are typically difficult to cool, and are subjected to potential damage due to high thermal loads. Blade tips at high temperatures may also wear out due to hard rubs against the shroud. Unshrouded blades have a gap existing between the blade tip and the shroud surface, which is known as tip gap. Blade tip failure is primarily caused by hot leakage flow through the tip gap. The leakage flow accelerates due to a pressure difference between both the pressure and suction sides of the blade, causing thin boundary layers and high heat transfer rates. This tip leakage flow is undesirable because it chips away the pressure side tip comer from mid-chord to trailing edge. As the blade tip is chipped away, the tip gap width increases allowing more leakage flow through the tip gap and accelerating blade tip failure. It has been recognized that the blade tip geometry and subsequent tip leakage flows have a significant effect on the aerodynamic efficiency of turbines. The influence of blade tip clearance on turbine efficiency is so significant that designers have a strong desire to improve efficiency by decreasing tip-to-shroud operating clearances, or by implementing more effective tip leakage sealing mechanisms. Whatever the design choice 
selected for a particular turbine blade tip, accurate and detailed knowledge of the flow field and heat transfer on the tip and the near-tip region is important for an efficient balanced airfoil design. Reliable experimental data are also important to develop and validate computational codes to predict blade metal temperatures, and an efficient cooling system design.

The existing literature contains many experimental and numerical investigations on flow field in and around turbine blade tip models. Studies by Bindon and Morphus (1988) and Bindon (1989) have contributed to the general understanding of tip leakage flow patterns. Bindon concluded that the growth of a vortex, which forms as the flow separates on the pressure side wall and exits through the tip gap causing an increase in the secondary flow, results in large aerodynamic losses. Moore et al. (1989) also contributed to the understanding of the flow field through tip gaps. Yaras and Sjolander (1991) studied the effect of simulated rotation on tip leakage and found a significant reduction in the gap mass flow rate due to rotation. Sjolander and Cao [1995] studied the flow field in an idealized turbine tip gap. They concluded that the flow separates from the pressure side tip comer and forms a well-organized chord-wise vortex above the blade tip. The separation vortex induces a flow back toward the pressure comer, resulting in the formation of a secondary counter-rotating vortex. Thus, the unexpected region of attached flow near the pressure corner may have a reasonably high convective heat transfer coefficient, in addition to the high wall shear stress. Kaiser and Bindon (1997) investigated a quantitative analysis of the effects of tip clearance, tip geometry, and multiple stages on turbine stage efficiency in a rotating turbine rig environment. Many other works studied the effect of tip clearances on leakage and efficiency loss prediction.

Turbine blade tip heat transfer is an important aspect that has drawn considerable attention from researchers in the past several years. The earliest study on blade tip heat transfer was done by Mayle and Metzger (1982). They studied heat transfer in a simulated 2-D rectangular tip with and without a rotating shroud. They concluded that the effect of blade rotation could be neglected to assess blade tip heat transfer. The pressure-driven flow through the tip gap (tip leakage) mainly influences the heat transfer. Metzger et al. (1989) and Chyu et al. (1989) studied heat transfer for both flat and grooved rectangular tip models. They incorporated the effect of relative motion by introducing a moving shroud surface over the grooved tip model. They affirmed that relative motion had little effect on the average tip heat transfer, though some local effects were observed.

Experimental data on blade tip heat transfer in a cascade environment are limited in the open literature. Yang and Diller (1995) reported local heat transfer coefficient on a turbine blade tip model with a recessed cavity in a stationary linear cascade. Based on a single heat flux sensor, they found the convection coefficients insensitive of tip gap height. Metzger et al. (1991) used several heat flux sensors and measured local tip heat fluxes on the flat tips at two different tip gaps in a rotating turbine rig. Their study was primarily confined to the blade forward region. The most recent study on turbine blade tip heat transfer and flow in a linear cascade was carried out by Bunker et al. (1999). Their experimental study investigated the detailed distribution of convective heat transfer coefficients on the first stage blade tip surface for a geometry typical of a large power generation turbine. This study provided a nearly full surface information on heat transfer coefficients in a non-rotating cascade environment with appropriate pressure distribution on the blade tip and shroud model. They used a three-blade cascade; however, only the center blade (test blade) had a variable tip gap clearance. The test airfoil was modeled after the aerodynamic tip section of a large power generation turbine. They used a hue detectionbased liquid crystal technique to obtain detailed heat transfer coefficient distributions on the blade tip surface for flat, smooth tip surfaces, with both sharp and rounded edges.

With the development of efficient numerical codes, some researchers carried out numerical investigations to predict blade tip heat transfer and flow field. Metzger et al. (1991) provided a numerical model to estimate tip and shroud heat transfer. Ameri and Steinthorsson $(1995,1996)$ predicted rotor blade tip and shroud heat transfer for a SSME (Space Shuttle Main Engine) turbine. Ameri et al. (1997) also predicted flow and heat transfer on the tip of a full rotating GE- $E^{3}$ first stage rotor blade for both a smooth tip and recessed tip. They found a separation vortex generated by the incoming flow separating off the inner edge of the pressure side rim and exiting near the trailing edge of the blade. For the heat transfer contours on the blade tip, they found a sharp entrance effect on the pressure side of the tip surface, where the heat transfer rate reached a maximum due to flow reattachment. The heat transfer contours on the flat tip also showed that a low heat transfer region existed near the leading edge suction side. The heat transfer rate near the trailing edge was lower, and the suction side had a lower heat transfer rate than the pressure side. Ameri et al. (1998) also predicted the effects of tip clearance and casing recess on heat transfer and stage efficiency for several squealer blade-tip geometries. Most recently, Ameri and Bunker (1999) performed a computational study to investigate detailed heat transfer distributions on blade tip surfaces for a large power generation turbine. They compared and validated their model with the experimental data of Bunker et al. (1999) obtained for the same geometry. However, there are very few experimental data available in the open literature to compare and validate numerical models. Without reliable experimental data, the numerical models could not be properly employed in the design and analysis of blade tip heat transfer and flow field.

This study will fulfill the need for additional heat transfer data on a gas turbine blade tip. The test section used for this study is a fivebladed linear cascade, with the three middle blades having a variable tip gap. The tip profile used here represents a first stage rotor blade tip of a modem aircraft gas turbine engine $\left(G E-E^{3}\right)$. Systematic pressure measurements in the near-tip region and on the shroud surface, and heat transfer measurements on the blade-tip surface are done for a tip gap clearance of $1 \%, 1.5 \%$, and $2.5 \%$ of the blade span. Two inlet freestream turbulence intensity levels are also considered. The effect of unsteady wakes, shock waves, and blade rotation, which may be important in real operating condition, is not considered in this study. This study provides a complete experimental pressure and heat transfer data on a gas turbine blade tip profile $\left(\mathrm{GE}-\mathrm{E}^{3}\right)$, whose profile geometry is open to the public domain. This data is presented in a standard format with standardized boundary conditions that could also be used by numerical people in the gas turbine community.

\section{EXPERIMENTAL SETUP}

The experimental setup is a stationary blow down facility with a five-bladed linear cascade. Air from a compressor enters a storage tank at about 280 psig. Then the air enters a high flow pneumatic control valve through a pneumatic ball valve. A controller, regulating the flow rate in downstream of the control valve, is set manually for the desired pressure. The controller gets feedback of the downstream pressure through a pressure line connected to the controller. The controller is capable of maintaining a steady downstream velocity within $\pm 3 \%$ of the desired set value. Downstream from the control valve, the test loop consists of a $5 \mathrm{~m}$ long circular duct with a $10.16 \mathrm{~cm}$ inside diameter and a $3.1 \mathrm{~m}$ long rectangular duct of $31.1 \times 12.2 \mathrm{~cm}$. A symmetric tapered section connects the two ducts. A by-pass flow path containing a rupture disk is also connected to the main flow circular duct to safeguard the test section against over-pressure. 


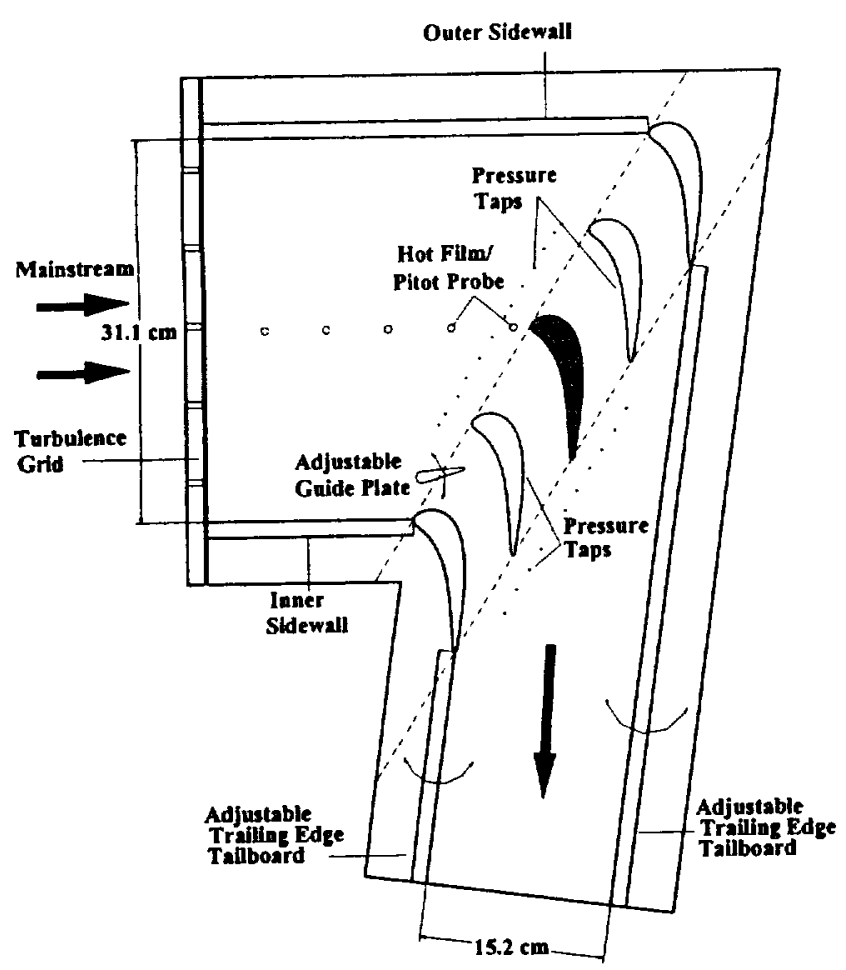

Fig. 1. Test Section with 5-Blade Cascade

The blade cascade is connected at the end of the rectangular duct. A small gap is maintained at the junction of the blade cascade and the rectangular duct to trip the boundary layer. This location for the boundary layer trip is $26.7 \mathrm{~cm}$ away from the center blade leading edge. A turbulence-generating grid of $57 \%$ porosity is also placed at this location to generate high turbulence for the test cases. The turbulence grid is composed of $12.25-\mathrm{mm}$ wide square bars with $33.02 \times 27.94 \mathrm{~mm}$ opening between bars. Hot film anemometry measurements, using a TSI IFA- 100 unit, show that the free-stream turbulence intensity level at a distance of $6 \mathrm{~cm}$ upstream from the test-blade leading edge is $6.1 \%$ without the turbulence grid and $9.7 \%$ with the turbulence grid. The turbulence length scale was estimated to be $1.5 \mathrm{~cm}$ for the $9.7 \%$ turbulence case, which is slightly larger than the grid size.

The test section is a five-bladed linear cascade with 4-flow passages as shown in Figure 1. The two far-end blades work as guide vanes and the outside wall, and the center blade work as a test blade. The cascade inlet dimensions are $31.1 \mathrm{~cm}$ wide and $12.2 \mathrm{~cm}$ high (span). Each blade has a $12.2 \mathrm{~cm}$ span and a $8.61 \mathrm{~cm}$ axial chord length. This dimension is three times $(3 \mathrm{X})$ the dimensions of a GE- $\mathrm{E}^{3}$ blade profile. The blades are made of aluminum and are EDM machine finished. The test section's top, bottom, and sides are made of $1.27 \mathrm{~cm}$ thick clear Polycarbonate (Lexan); however, a $1.2 \mathrm{~cm}$ thick clear acrylic replaces the top cover plate (shroud) for heat transfer tests, to facilitate the best optical access to the test blade (center blade). Two separate but identical blades are used for both the pressure and heat transfer measurements.

Figure 2 represents the blade tip configuration in the cascade. Each blade has a constant cross section for the entire span and represents the tip section of an aerodynamic turbine blade. The blade leading edge pitch is $9.15 \mathrm{~cm}$ and the axial chord length is $8.61 \mathrm{~cm}$. The throat diameter at the point of minimum distance between two blades is $4.01 \mathrm{~cm}$, which, with a span of $12.2 \mathrm{~cm}$, gives a throat aspect ratio of about 3 . The inlet flow angle to the test blade is $32.01^{\circ}$ and the exit angle is $65.7^{\circ}$, giving a total tuming of $97.71^{\circ}$. A variable tip gap

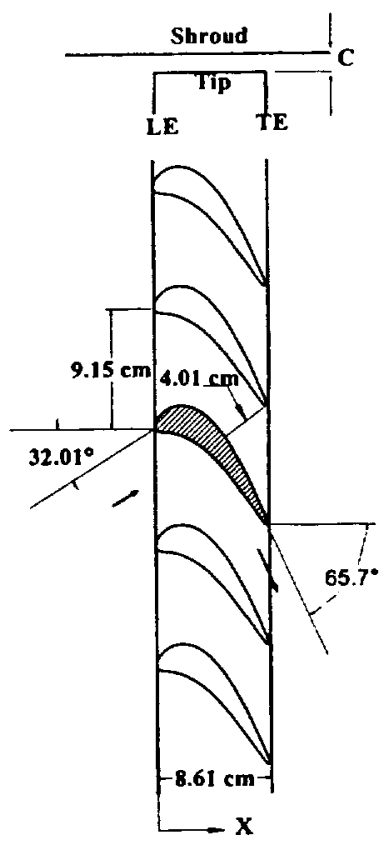

Fig. 2. Blade Tip and Shroud Definition

(C) is maintained between both the tip and shroud surfaces as shown in Figure 2. The tip gaps used for this study are $1.31 \mathrm{~mm}, 1.97 \mathrm{~mm}$, and $3.29 \mathrm{~mm}$, which correspond to about $1 \%, 1.5 \%$, and $2.5 \%$ of the blade span $(12.2 \mathrm{~cm})$. Hard rubber gaskets of desired thickness are placed on top of the sidewalls, the trailing edge tailboards, and the two outer guide blades to create tip gaps of desired height.

Figure 3 shows the pressure tap blade configuration, which is made of aluminum with a set of pressure taps. Each pressure tap tubing has an outer and inner diameter of $1.65 \mathrm{~mm}$ and $1.35 \mathrm{~mm}$, respectively. The tap holes are located in the mid-span ( $50 \%$ of blade height) of the blade, at a height of $80 \%, 90 \%$, and $97 \%$ of the span as measured from the base of the blade. The pressure taps are placed on both the pressure

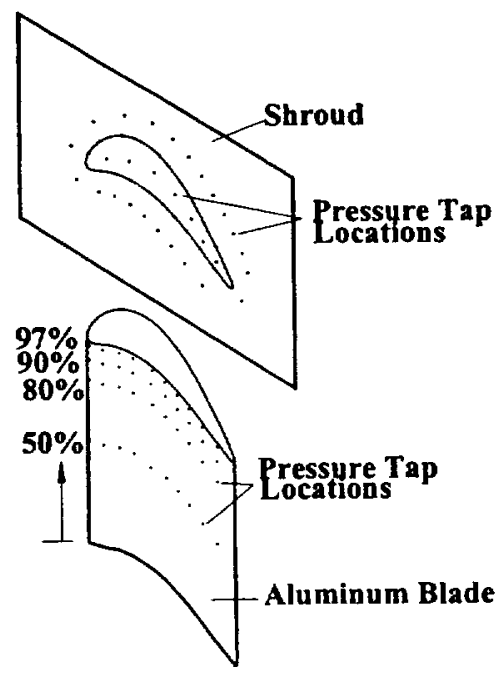

Fig. 3. Pressure Tap Locations on Blade and Shroud 
and suction surfaces. Pressure taps are also placed on the shroud surface, as shown in Figure 3, to measure the pressure distribution on the shroud surface, which is a representative of the tip surface pressure. A total of 52 pressure taps measure the shroud surface pressure. One set of pressure taps is located around the tip perimeter and along the mean camber line, and a second set of taps is located $12.52 \mathrm{~mm}$ outside the tip edge. All these pressure taps are located on the shroud surface opposite the tip surface. No pressure taps are placed on the tip surface. These pressure distributions are useful in estimating the tip leakage flow.

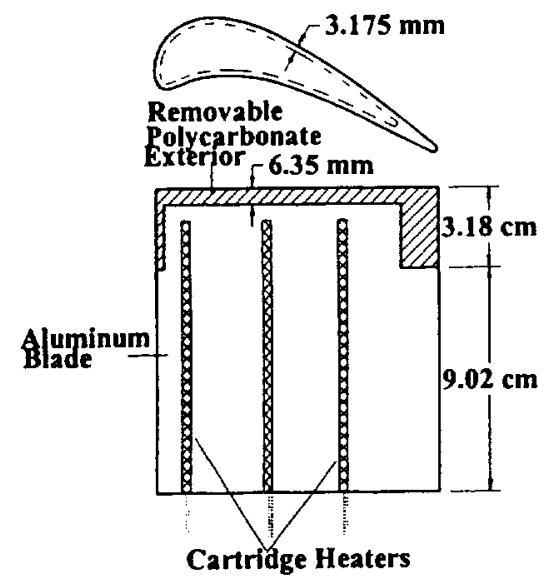

Fig. 4. Heat Transfer Blade

The heat transfer blade, as shown in Figure 4, is designed with a specific feature. The lower portion of the blade is made of aluminum for structural rigidity against the aerodynamic forces present during the tests. The upper portion of the blade has an inner aluminum core and an outer shell made of black polycarbonate with a low value of thermal conductivity for transient liquid crystal test. The base thickness of the polycarbonate shell is $6.35 \mathrm{~mm}$ and the wall thickness is $3.175 \mathrm{~mm}$. It is closely fitted with the inner aluminum core. The shell is also glued to the inner core through the rim contact surface for better rigidity. Three cartridge heaters are embedded into the inner core. The cartridge heater provides heating to the aluminum core, which in turn, heats the outer polycarbonate shell. The blade is fastened to the bottom endwall with screws.

The usual operating condition for this cascade is set at an inlet total pressure of $143 \mathrm{kPa}$, an exit average static pressure of $108.3 \mathrm{kPa}$, which gives an overall blade pressure ratio of 1.32. The mass flow rate (up to 60 second) through the cascade is about $5.9 \mathrm{~kg} / \mathrm{s}$. During the blow down test, the inlet air velocity is kept at about $85 \mathrm{~m} / \mathrm{s}$; the exit air velocity at $199 \mathrm{~m} / \mathrm{s}$, and the corresponding Reynolds number of $1.1 \times 10^{6}$ based on axial chord length and exit flow velocity. The corresponding Mach numbers at the inlet and exit are 0.25 and 0.59 , respectively.

\section{FLOW CONDITION IN THE CASCADE AND PRESSURE MEASUREMENT}

To check the flow condition in the cascade, the blades next to the suction and pressure sides of the center (test) blade are instrumented with pressure taps with static pressure holes at mid-span of the blades (as shown in Figure 1). The suction surface of the inner-side blade has six pressure taps and the pressure surface of the outer-side blade has five pressure taps, each at $50 \%$ height of blade span. Pressure taps are also instrumented at 13 locations at the inlet and 11 locations at the exit plane, as shown in Figure 1. These inlet and exit plane taps are located at a distance of $25 \%$ of the blade span upstream from the blade leading edge, and downstream from the blade trailing edge, respectively. The cascade has adjustable trailing edge tailboards. A thinner, adjustable guide plate is also placed near the inlet between the passage of the two interior blades in the shorter flow passage side as shown in Figure 1. This guide plate and the adjustable tailboards help provide an identical flow through the pressure and suction side passages of the test blade. With no adjustment, a substantially greater percentage of the total flow would go through the shorter flow passage. The guide plate is rounded on the leading side and tapered and rounded on the trailing side to minimize flow disturbances. A final position for both the adjustable guide plate and trailing edge tailboards is selected after many variations have been tried.

The inlet and exit flow pattern is represented by the coefficient of pressure $\left(C_{p}\right)$. The coefficient of pressure is defined as:

$$
C_{p}=\frac{P_{t}-P}{P_{t}-P_{a v g}}
$$

Here, $P_{a v g}$ is the average static pressure as measured by the inlet (or exit) plane pressure taps, $P_{t}$ is the total inlet pressure, and $P$ is the local static pressures as measured by the inlet (or exit) pressure taps. This $C_{p}$ actually represents a non-dimensional velocity field, which is important when quickly reviewing the velocity field pattem. Figures $5 \mathbf{a}$ represents the $C_{p}$ at the cascade inlet and the exit plane. The axial distance is measured from the outermost tap location towards the innermost tap (cascade outer and inner sidewalls are labeled in Figure 1). Figure $5 b$ shows pressure distributions at a $50 \%$ height of the blade span on the center blade and the two nearby blades. The local axial positions are normalized by the axial chord length. The multiple data points represent the variation in repeated tests. The result shows that the flow patterns in the two passages are almost identical.

Static pressure measurements are also made at $80 \%, 90 \%$, and $97 \%$ height of the blade span on both the P/S and S/S, and also on the shroud surface opposite the blade tip surface. A total of 52 pressure taps measured the shroud surface pressure. One set of pressure taps is located around the tip perimeter and along the mean camber line, while a second set of taps is located $12.52 \mathrm{~mm}$ outside the tip edge. All these pressure taps are located on the shroud surface opposite the tip surface. Pressures are recorded with a 48-channel Scanivalve System coupled with LabView 5.0 software. LabView discarded all data that fell outside the initial mean \pm 1.5 standard deviation. It then recorded the mean value of the screened data. The computer steps through each tap and measures the local static pressure on the blade suction and pressure surfaces, and the shroud. Every pressure measurement is repeated at least three times to reduce operating uncertainty and to verify the repeatability of the data. The blow down facility is capable of giving a required flow for about one minute. The control valve and controller are capable of providing a steady flow condition (the velocity variation is within $\pm 3 \%$ ) in this one minute time period. Figure 6 shows the ratio of the total to local static pressure distributions on the $P / S$ and the $\mathrm{S} / \mathrm{S}$ at different blade span heights for $1.97 \mathrm{~mm}$ (1.5\% of the span) tip gap and inlet $T u=6.1 \%$ only. The ratio of pressure distribution $\left(P_{t} / P\right)$ is presented as a function of normalized axial distance $\left(X / C_{x}\right)$. A higher value of $P_{k} / P$ corresponds to a lower static pressure, while a lower value corresponds to a higher static pressure. The static pressure difference between the $\mathrm{P} / \mathrm{S}$ and the $\mathrm{S} / \mathrm{S}$ is the main driving force for the leakage flow. Figure 6 clearly shows that the maximum static pressure- 


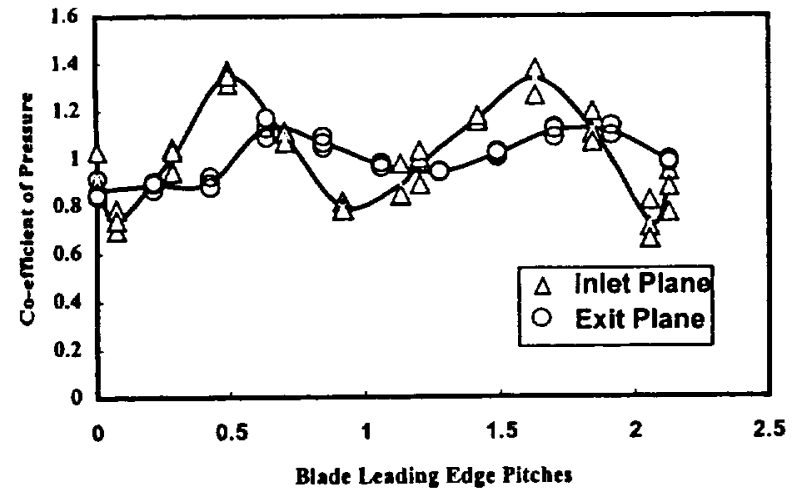

Fig. 5a. Co-efficient of Pressure at Inlet and Exit Plane

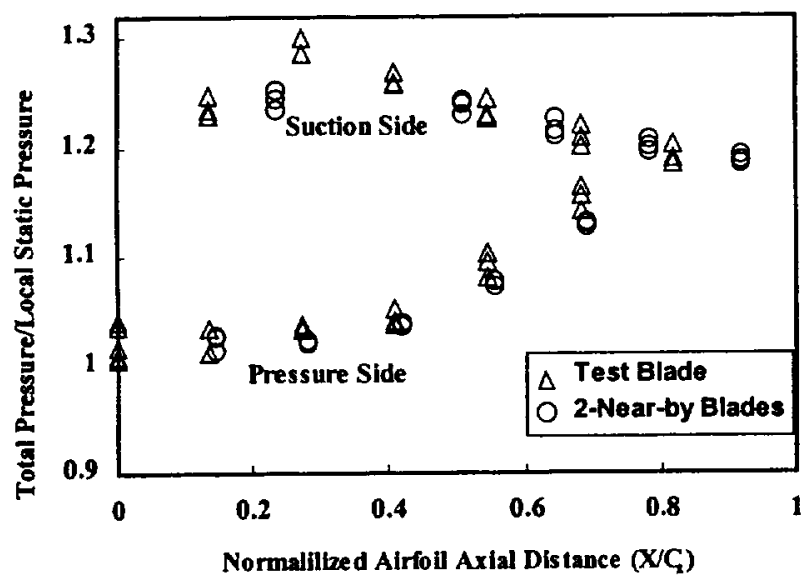

Fig. 5b. Pressure Dis tribution at Mid-span on the Test Blade and 2-Near-by Blades

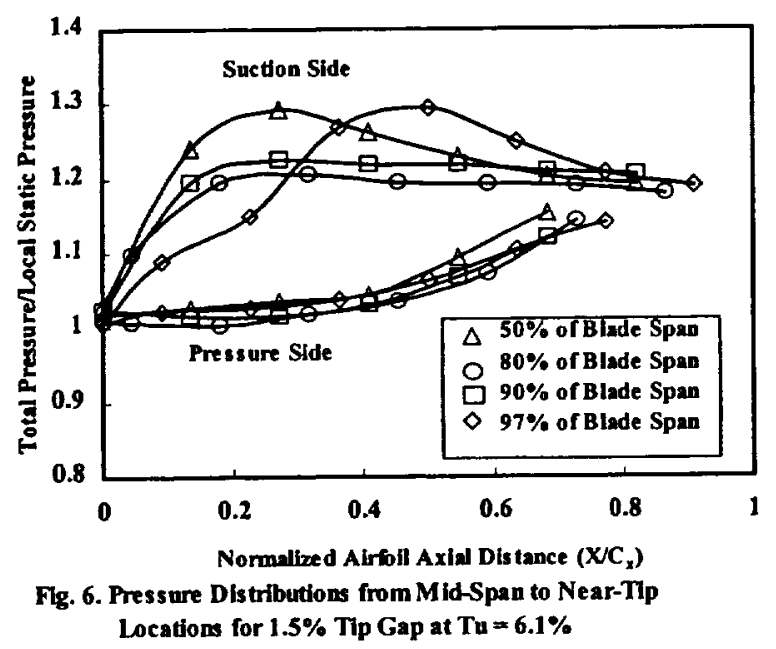

difference occurs at a distance of $20-30 \%$ of the axial chord from the leading edge at $50 \%$ of the blade span. This location of maximum pressure difference has shifted toward the TE at $97 \%$ of the blade span, and the maximum static pressure difference occurs at about $50 \%$ of the axial chord from the LE. This shift is due to the leakage flow through the tip gap.

Figures 7 shows the total pressure to local static pressure ratio $\left(P_{1} / P\right)$ contours on the shroud surface for three different tip gaps at $\mathrm{Tu}=$
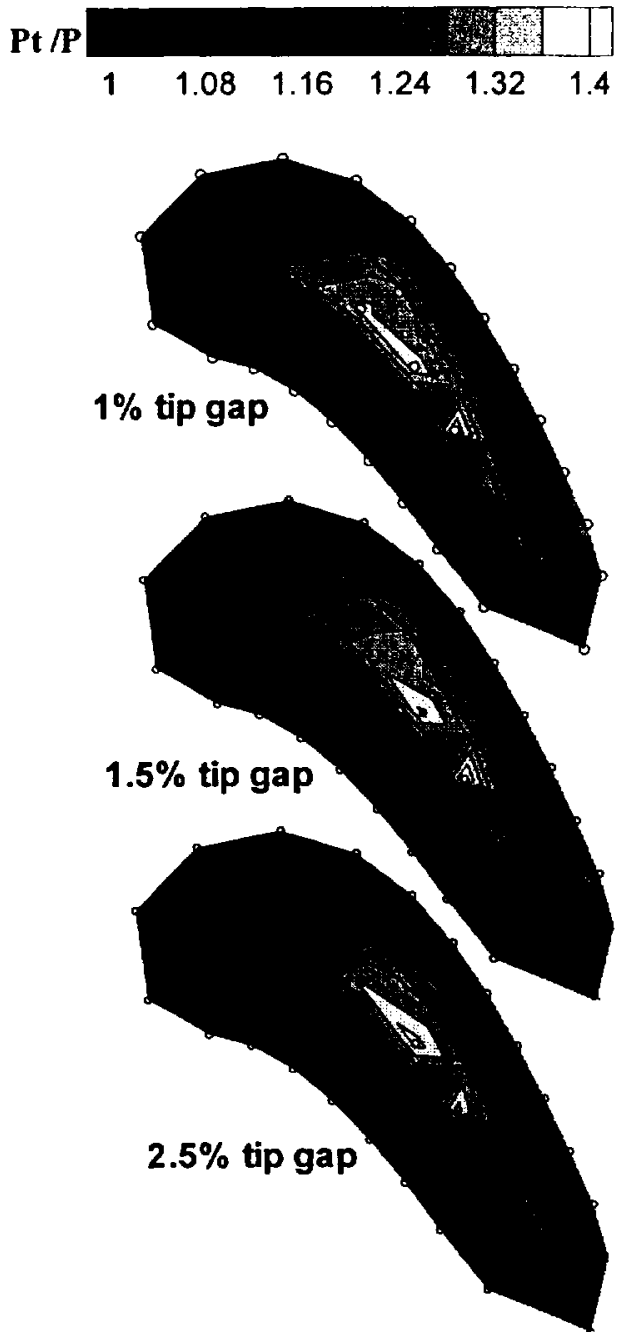

Fig. 7. Pressure Ratio Distribution (Pt/P) on Shroud Surface

$6.1 \%$. This contour plot helps explain the heat transfer results on the tip surface. The small circles on the contour plots represent the pressure tap locations relative to the tip, while the bold line indicates the tip dimension. A smaller $P_{\mathbb{V}} / \mathrm{P}$ value means higher static pressure, while a larger value means a lower static pressure. This pressure ratio distribution clearly demonstrates the tip leakage flow path. The lower $P, P$ value on the $P / S$ LE indicates that the leakage flow enters the tip gap at this location, while the higher $P t / P$ value on the $S / S$ indicates that the leakage flow exits the tip through this location. In this flow path, a separation vortex generates as the incoming flow separates off the inner edge of the P/S rim and exits near the S/S TE of the blade. Sjolander and Cao (1995) observed this separation vortex and Ameri et al. (1997) also predicted this phenomenon. Due to this separation vortex, the heat transfer rate increases in this region as seen in the heat transfer results.

Figure 7 also shows that as the tip gap decreases, the $P / P$ ratio slightly increases toward the LE $S / S$, resulting in a change in the leakage flow path direction. This means, that the tip leakage flow path direction is shifting towards the LE S/S at a lower tip gap. This happens because, at lower tip gap, the leakage flow has a smaller gap to flow through, over the boundary layer that develops at the P/S and grows toward the $\mathrm{S} / \mathrm{S}$. Thus, the leakage flow has to overcome higher resistance compared to a higher tip gap situation. As a result, it shifts 
the flow direction toward a shorter path. This effect is reflected in the heat transfer results. Pressure measurements are not made on the blade tip surface. However, Bunker et al. (1999) showed that the pressure distribution trends are very similar at both the blade tip and shroud surfaces.

\section{HEAT TRANSFER MEASUREMENT AND RESULTS}

Heat transfer measurement is made at a preset flow condition at which the pressure measurement is done. A threshold intensity method of transient liquid crystal technique is used. The liquid crystals in this study are wide band 30 to $35 \mathrm{C}$ crystals made by Hallcrest (R30C5W). The blade tip surface is initially coated with a thin layer of thermochromic liquid crystals, and the blade is fastened in the test cascade. The cartridge heaters are turned on for three hours. The cartridge heaters heat the inner aluminum core of the test blade and the aluminum core, in tum, heats the polycarbonate shell to a desired steady initial temperature of about $60 \mathrm{C}$. The initial tip surface temperature is measured using twenty two (22) thermocouples placed on the blade tip surface at different discrete locations. Two thermocouples are also placed on the suction and pressure side near-tip region toward the trailing edge. These two thermocouples work as a reference to check the initial tip surface temperature during the heat transfer test. This process of heating the blade and measuring the initial temperature is repeated several times before the final heat transfer test to ensure repeatability of the recorded initial temperature. The initial temperature is very uniform (within $2 \mathrm{C}$ ) throughout the tip surface, except near the trailing edge region. These temperatures are then interpolated to get an initial temperature map on the whole surface.

The thermocouples from the tip surface are removed, the blade is then washed and re-sprayed with liquid crystals. The blade is put back in the cascade and the cartridge heaters are turned on for three hours. A region of interest (ROI) is selected and the blow down test is then carried out. This region of interest (ROI) is the data collection region. The image processing system captures the liquid crystal color change time from green to the onset of red during the blow down test. The image system requires a background intensity correction based upon the lighting conditions on the ROI. This is called threshold, which ensures that all the points in the ROI are at a lower intensity than the real greento-red transition intensity caused by liquid crystal color change. The system is ready for the blow down test once the lighting, threshold, and initial temperature are set. Only one test is done per day in a controlled environment (test cell doors are closed and the room temperature is controlled). The reference thermocouples check the initial surface temperature during a heat transfer test. Precise coordination of the image processing system and the flow is critical, since the heat transfer experiments are performed at transient conditions. Thermocouples placed at the cascade inlet provide the free-stream temperature, which is about $24 \mathrm{C}$. The blade surface color change is monitored using an image processing system. The image processing system consists of a high resolution RGB color CCD camera, a high speed PC with a 24-bit true color frame grabber board (from Imaging Technology), and a Color Video Monitor with RGB inputs. The camera captures real-time images through Optimas 3.0, an image processing software. The same software translates the captured image into a data file. The test duration is small enough $(\sim 10-30 \mathrm{sec})$ to consider a semi-infinite solid assumption. The local heat transfer coefficient on the blade tip surface is then calculated, assuming a one-dimensional transient conduction into a semi-infinite solid surface with a convective boundary condition using the following equation:

$$
\frac{T_{w}-T_{i}}{T_{\infty}-T_{i}}=1-\exp \left(\frac{h^{2} \alpha t}{k^{2}}\right) \operatorname{erfc}\left(\frac{h \sqrt{\alpha t}}{k}\right)
$$

Here, $T_{w}(30 \mathrm{C}), T_{i}(60 \mathrm{C}), T_{\propto}(24 \mathrm{C}), \mathrm{t}(-10-30 \mathrm{sec}), \mathrm{k}(0.18 \mathrm{~W} / \mathrm{m} \mathrm{K})$, and $\alpha$ are known. The local heat transfer coefficient at any location can then be calculated from the above equation. The experimental uncertainty is measured using the methods of Kline and McClintock (1953). The uncertainty of the local heat transfer coefficient measured by this method is estimated to be $\pm 7.9 \%$ or less. The uncertainty estimation does not include the effect of 2-dimensionality near the edges. Note that the acrylic blade material (polycarbonate) has a very low thermal conductivity of $0.18 \mathrm{~W} / \mathrm{m} \mathrm{K}$. The liquid crystal color change transition occurs at the surface, which is kept at a uniform initial temperature. Test duration is also smaller $(-10-30 \mathrm{sec})$ than the time required for the temperature to penetrate the full thickness of the insulating acrylic material. Thus, a 1-D transient, semi-infinite solid assumption is valid throughout the surface, except near the tip edges. Due to this 1-D assumption, the results at the tip edges are less reliable, and may suffer more uncertainty than the reported value because of the existing two-dimensional conduction effect.

The heat transfer coefficient measurements are done for the three cases of tip gaps and at the two different turbulence intensity levels. Figure $8 \mathrm{~b}$ shows the tip local heat transfer contour plots for a tip clearance gap of $1.97 \mathrm{~mm}$ (1.5\% of the blade span) and an inlet free stream turbulence intensity level of $6.1 \%$. This is a typical heat transfer coefficient distribution contour plot for each case tested here. The distribution clearly shows various regions of low and high heat transfer coefficient on the tip surface. The magnitude of the heat transfer coefficient varies from 500 to $1700 \mathrm{~W} / \mathrm{m}^{2} \mathrm{~K}$ on the tip surface. A surprisingly important finding is the development of a low heat transfer region near the $\mathrm{LE}$ and toward the $\mathrm{S} / \mathrm{S}$.
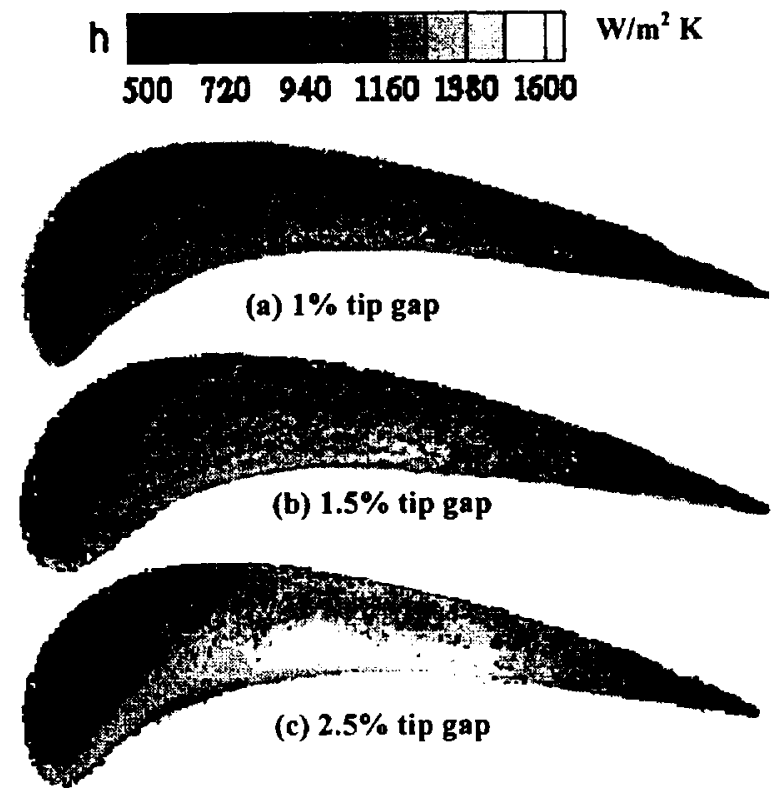

Fig. 8. Heat Transfer Coefficient at $\mathrm{Tu}=6.1 \%$

The pressure ratio $(P, P)$ distributions on the shroud surface (Figure 7) show that this low heat transfer region corresponds to the lowest convective velocity due to a lower static pressure difference $(\Delta \mathrm{P})$ between the pressure and the suction sides. A gradual increase in the heat transfer coefficient is observed surrounding the low heat transfer region. The tip leakage flow originates from the pressure side near the leading edge and exits through the suction side, away from the leading edge, as seen in Figure 7 . This region of maximum tip leakage flow 
corresponds to a high heat transfer coefficient as seen in Figure $\mathbf{8 b}$. This means that the higher heat transfer coefficient is observed along the tip leakage flow path. The heat transfer coefficient along this tip leakage flow path is higher toward the pressure side than the suction side. This happens because of the flow entrance effect. The leakage flow enters through the pressure side and exits through the suction side. A separation vortex generates at the pressure side as the incoming flow separates off the inner edge of the pressure side rim and exits near the trailing edge of the blade. Sjolander and Cao (1995) observed this separation vortex and they mentioned about the possibility of a high heat transfer rate on the pressure side. The lower heat transfer coefficient toward the suction side may be the result of a boundary layer that develops at the pressure side edge and grows toward the suction side. Figure $\mathbf{8 b}$ also shows a lower heat transfer coefficient near the trailing edge region. This region corresponds to a lower tip leakage flow (lower $\triangle \mathrm{P}$ ) as seen in Figure 7, thus, resulting in a lower heat transfer coefficient.

Bunker et al (1999) found a similar trend of low and high heat transfer region on the tip surface. They termed the low heat transfer region as the "sweet spot". They found that the low heat transfer region originates from the airfoil pressure side at about 20 to $30 \%$ axial chord location, while we found this location near the LE S/S. This spatial difference may be due to a difference in the tip model geometric profile, and orientation of the blade with respect to the flow path. They used a tip model geometry of a large power generation turbine, while ours is an $\mathrm{E}^{3}$ profile of an aircraft gas turbine engine. Note that, the level of the local heat transfer coefficient on our $E^{3}$ blade and on their power generation turbine is of the same order of magnitude. Ameri et al. (1997) predicted heat transfer rate on the tip of a full rotating $E^{3}$ blade. The trend of their predicted result is very similar to our experimental data. They also predicted a low heat transfer region near the leading edge suction side, while the heat transfer rate is higher on the pressure side and lower near the trailing edge as seen in our case.

Figure $8 \mathrm{a}$ and $\mathrm{c}$ show the tip heat transfer coefficient distribution for $1.31 \mathrm{~mm}$ ( $1 \%$ of the span) and $3.29 \mathrm{~mm}$ ( $2.5 \%$ of the span) tip gaps, respectively at a same inlet turbulence intensity level of $6.1 \%$. These figures clearly show that the tip gap has a significant effect on the local heat transfer coefficient. A larger tip gap results in an overall higher heat transfer coefficient, while a smaller tip gap results in a lower heat transfer coefficient. This is because a larger tip gap increases the amount of tip leakage flow, while a smaller tip gap decreases it. However, the local heat transfer coefficient changes according to a change in the leakage flow path direction. Figure 8 shows that a decrease in the tip gap from $1.5 \%$ to $1 \%$ decreases the tip local heat transfer coefficient distribution by about $10 \%$ at high heat transfer region along the flow path, while slightly increases the local heat transfer coefficient in the low heat transfer region. Similarly, an increase in the tip gap from $1.5 \%$ to $2.5 \%$ increases the local heat transfer coefficient by about $20 \%$ in the high heat transfer region, while slightly decreases in the low heat transfer region. It happens, because the tip leakage flow path slightly changes its direction depending on the gap size. At a larger tip gap, the leakage flow can easily pass toward the trailing edge suction side overcoming the boundary layer thickness. Whereas, at a smaller tip gap, the leakage flow encounters more resistance to the flow toward the trailing edge suction side due to a smaller passage over the boundary layer thickness. Thus, the flow shifts its direction toward a shorter passage in the leading edge suction side, which results in a higher heat transfer coefficient in this region. The shroud pressure distribution in Figure 7 supports this phenomenon.

Figures $9 \mathbf{a}, \mathbf{b}$, and $\mathbf{c}$ show the heat transfer coefficient distributions for $1.31 \mathrm{~mm}(1 \%), 1.97 \mathrm{~mm}(1.5 \%)$, and $3.29 \mathrm{~mm}(2.5 \%)$, respectively at a higher inlet turbulence intensity level of $9.7 \%$. The heat transfer distribution trend is the same as that of Fig $\mathbf{8 a}, \mathbf{b}$, and $\mathbf{c}$ at a lower turbulence level of $6.1 \%$. However, the magnitude of the overal!
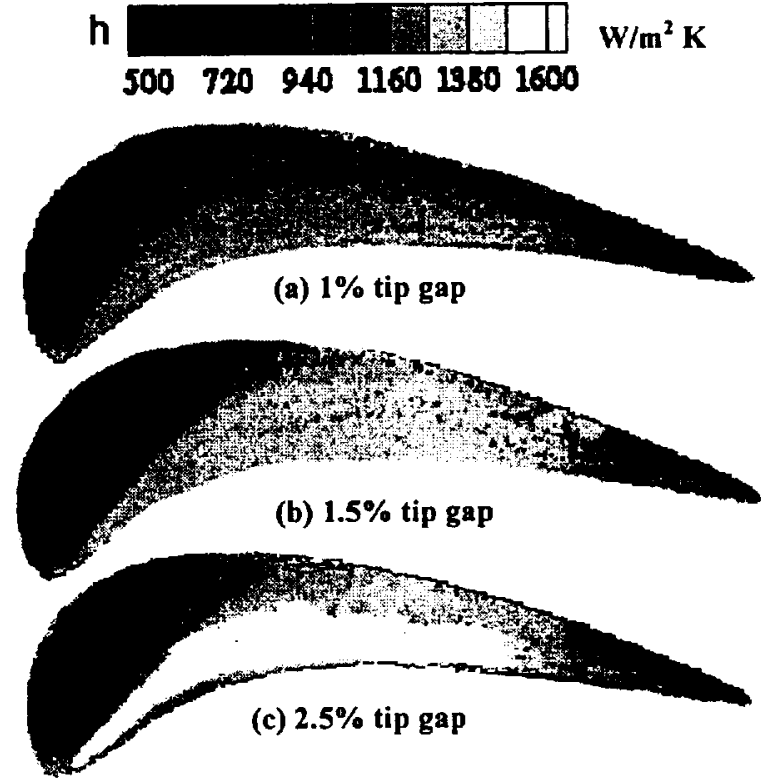

Fig. 9. Heat Transfer Coefficient at Tu=9.7\%

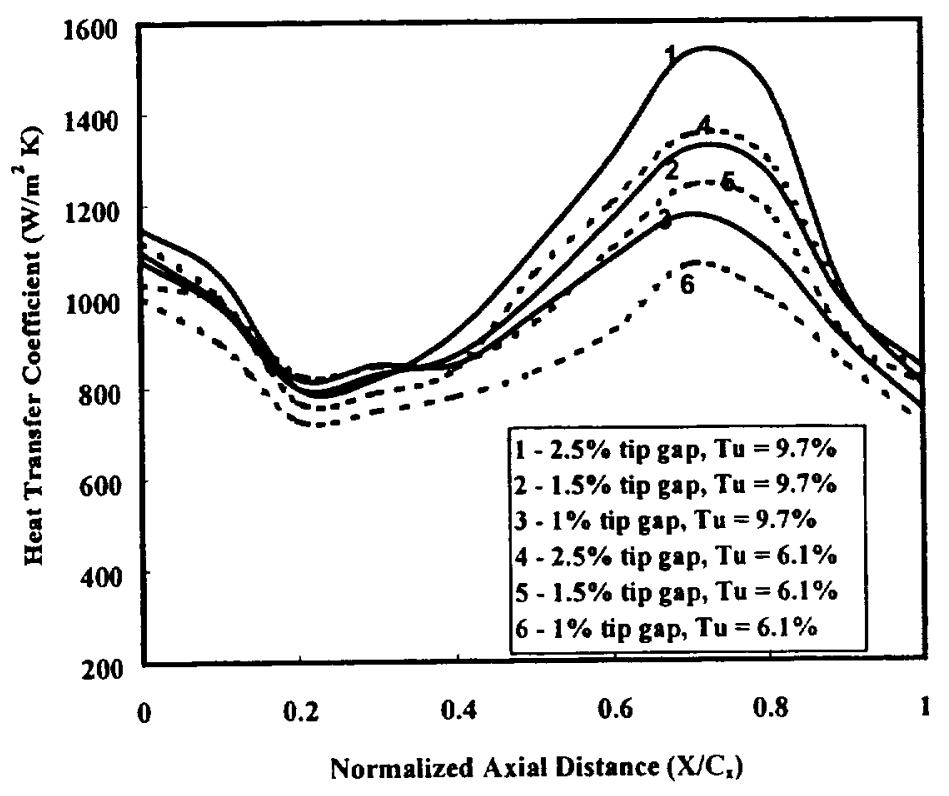

Fig. 10. Averaged Heat Transfer Coefficient

heat transfer coefficient is about $15-20 \%$ higher over the low turbulence case of $6.1 \%$ intensity level. The increase in heat transfer coefficient is more prominent along the leakage flow path, because turbulence does not increase the magnitude of the tip leakage flow; however, it increases flow fluctuations.

The averaged tip heat transfer coefficients for the three different tip gaps and for both the low and high turbulence cases are presented in Figure 10. The result shows that, for each case of the tip gap, the heat 
transfer coefficient initially decreases along the axial chord length. It then increases and becomes a maximum at about $70 \%$ location of the axial chord, and again decreases toward the trailing edge. Smaller tip gaps produce a lower averaged tip heat transfer coefficient. Similar effect of the tip gap is observed at both the lower and higher turbulence levels of $6.1 \%$ and $9.7 \%$, while the magnitude of heat transfer coefficient is higher at higher turbulence case of $9.7 \%$ compared to the low turbulence case of $6.1 \%$.

\section{CONCLUSIONS}

This study investigated the effect of tip gap and inlet turbulence intensity on detailed local heat transfer coefficient distribution on the plane tip surface of a gas turbine blade. The blade tip model is a 2 dimensional profile of an $E^{3}$ blade of an aircraft gas turbine engine. A transient liquid crystal technique is used for detailed heat transfer measurements. Pressure distributions in the near tip region and on the shroud surface provide complementary information explaining the local heat transfer behavior on the tip surface in a five-bladed stationary linear cascade. A typical operating condition having a Reynolds number based on airfoil axial chord and an exit velocity of $1.1 \times 10^{6}$ and an overall pressure ratio of 1.32 is used to measure the pressure and heat transfer coefficients. The major findings are:

1) Detailed measurements provide a better understanding of the local heat transfer behavior on the blade tip surface.

2) The pressure measurements in the near tip and on the shroud surface provide complementary information of the tip leakage flow pattern. This pressure data provides a basis for determining the tip leakage flow and also explains the heat transfer results. This detailed pressure and heat transfer measurement also provides a reference for further experimental or computational study.

3) Different heat transfer regions exist on the tip surface. The leading edge side contains a low heat transfer region near the suction side, while a high heat transfer region exists along the leakage flow path.

4) A higher heat transfer coefficient exists on the tip surface toward the pressure side because of the entrance effect.

5) Generally, a larger tip gap results in a higher overall heat transfer coefficient, while a smaller tip gap results in a lower overall heat transfer coefficient. This is because a larger tip gap increases the magnitude of the tip leakage flow, while smaller tip gap decreases it.

6) An increase in the inlet turbulence intensity level from $6.1 \%$ to $9.7 \%$ increases the heat transfer coefficient by about $15-20 \%$ along the leakage flow path.

\section{ACKNOWLEDGEMENT}

This work is prepared with support of the NASA Glenn Research Center under grant number NAG3-2002. The NASA technical team is Mr. Robert Boyle and Dr. Raymond Gaugler. Their support is greatly appreciated. Technical discussions with Dr. C. Pang Lee of GE Aircraft Engines, Dr. Ron Bunker of GE R\&D Center, and Dr. Srinath Ekkad of Louisiana State University were helpful and are acknowledged. Dr. C. Pang Lee also provided us with the $\mathrm{E}^{3}$ profile for the plane and squealer tips. His help is also appreciated.

\section{REFERENCES}

Ameri, A.A. and Bunker, R.S., "Heat Transfer and Flow on the First Stage Blade Tip of a Power Generation Gas Turbine: Part 2: Simulation Results," ASME 99-GT-283.

Ameri, A.A. and Steinthorsson, E., 1995, "Prediction of Unshrouded Rotor Blade Tip Heat Transfer," ASME 95-GT-142.
Ameri, A.A. and Steinthorsson, E., 1996, "Analysis of Gas Turbine Rotor Blade Tip and Shroud Heat Transfer," 96-GT-189.

Ameri, A.A., Steinthorsson, E. and Rigby, L. David, 1997, "Effect of Squealer Tip on Rotor Heat Transfer and Efficiency," ASME 97-GT128

Ameri, A.A., Steinthorsson, E. and Rigby, L. David, 1997, "Effects of Tip Clearance and Casing Recess on Heat Transfer and Stage Efficiency in Axial Turbines," ASME 98-GT-369.

Bindon, J.P., 1989, "The Measurement and Formation of Tip Clearance Loss," ASME Journal of Turbomachinery, Vol. 111, pp. 258263.

Bindon, J.P. and Morphus, G., 1988, "The Effect of Relative Motion, Blade Edge Radius and Gap Size on the Blade Tip Pressure Distribution in an Annular Turbine Cascade with Clearance," ASME 88-GT-256.

Bunker, R.S., Baily, J.C. and Ameri, A.A., 1999, "Heat Transfer and Flow on the First Stage Blade Tip of a Power Generation Gas Turbine: Part 1: Experimental Results," ASME 99-GT-169

Chyu, M.K., Moon, H.K. and Metzger, D.E., 1989, "Heat Transfer in the Tip Region of Grooved Turbine Blades," Joumal of Turbomachinery, Vol. 111, pp. 131-138.

Kaiser, I. and Bindon, J.P., 1997, "The Effect of Tip Clearance on the Development of Loss Behind a Rotor and a Subsequent Nozzle," ASME 97-GT-53.

Kline, S.J. and McClintock, F.A., 1953, "Describing Uncertainties in Single Sample Experiments," Mechanical Engineering, Vol. 75, pp. 3-8.

Mayle, R.E. and Metzger D.E., 1982, "Heat Transfer at the Tip of an Unshrouded Turbine Blade" Proc. Seventh Int. Heat Transfer Conf., Hemisphere Pub., pp 87-92.

Metzger, D.E., Bunker, R.S. and Chyu, M.K., 1989, "Cavity Heat Transfer on a Transverse Grooved Wall in a Narrow Flow Channel," Journal of Heat Transfer, Vol. 111, pp. 73-79.

Metzger, D.E., Dunn, M.G. and Hah, C., 1991, "Turbine Tip and Shroud Heat Transfer," Journal of Turbomachinery, Vol. 113, pp. 502507.

Moore, J., Moore, J.G., Henry, G.S. and Chaudhury, U., 1989, "Flow and Heat Transfer in Turbine Tip Gaps," Joumal of Turbomachinery, Vol. 111, pp. 301-309.

Sjolander, S. A. and Cao, D., 1995, "Measurements of the Flow in an Idealized Turbine Tip Gap," Journal of Turbomachinery, Vol. 117, pp. 578-584.

Yang, T.T. and Diller, T.E., 1995, "Heat Transfer and Flow for a Grooved Turbine Blade Tip in a Transonic Cascade," ASME-95WA/HT-29.

Yaras, M.I. and Sjolander, S.A., 1991, "Effects of Simulated Rotation on Tip Leakage in a Planar Cascade of Turbine Blades, Part ITip Gap Flow," ASME 91-GT-127. 